Z Rheumatol 2020 · 79:906-909

https://doi.org/10.1007/s00393-020-00842-y

Online publiziert: 23. Juli 2020

(c) Der/die Autor(en) 2020

\section{Redaktion}

M.O. Becker, Zürich

P. Hoff, Berlin

A.J. Hueber, Bamberg

F. Moosig, Neumünster

\author{
C. Thibeault ${ }^{1} \cdot$ U. Schneider ${ }^{2} \cdot$ A. Eisenschenk ${ }^{3} \cdot$ M. Lautenbach $^{3,4}$ \\ ${ }^{1}$ Medizinische Klinik mit Schwerpunkt Infektiologie und Pneumologie, Charité - Universitätsmedizin \\ Berlin, Campus Virchow Klinikum, Berlin, Deutschland \\ ${ }^{2}$ Medizinische Klinik mit Schwerpunkt Rheumatologie und Klinische Immunologie, Charité - \\ Universitätsmedizin Berlin, Campus Mitte, Berlin, Deutschland \\ ${ }^{3}$ Hand- und funktionelle Mikrochirurgie, Universitätsmedizin Greifswald, Greifswald, Deutschland \\ ${ }^{4}$ Abteilung Handchirurgie, obere Extremität und Fußchirurgie, Krankenhaus Waldfriede Berlin, Berlin, \\ Deutschland
}

\title{
Rezidiv einer septischen Arthritis des Handgelenks durch Pseudomonas aeruginosa bei Besiedelung eines zentral- venösen Verweilkatheters
}

lung und Synovialektomie sowie antibiotisch erfolgt. Bei Aufnahme war der Patient nach erfolgter Punctio sicca des betroffenen Handgelenks bereits auswärtig ambulant unter dem Verdacht einer Arthritis urica mit Prednisolon $50 \mathrm{mg} / \mathrm{Tag}$ sowie Diclofenac und Metamizol anbehandelt worden, worunter keine Besserung erzielt wurde. Er nahm außerdem dauerhaft Metformin, ASS, ein Statin und einen Betablocker ein.

\section{Befund}

\section{Klinische Untersuchung}

Bei der körperlichen Untersuchung fiel ein schmerzhaftes, gerötetes, überwärmtes und geschwollenes rechtes Handgelenk mit Ausbreitung der Schwellung über den Handrücken auf (• Abb. 1). Die Bewegungsmaße im Handgelenk waren schmerzbedingt in Extension und Flexion eingeschränkt (nach Neutral-NullMethode 20/0/20), der Faustschluss aller Finger inkomplett. Der Daumen war schmerzbedingt endgradig bewegungseingeschränkt. Der Patient war fieberfrei. Der Narbenbereich des einliegenden venösen Ports war klinisch unauffällig.

\section{Sonographie und Punktat des Ergusses}

Sonographisch zeigte sich ein minimaler Erguss des betroffenen Handgelenks. Das Punktat war leukozytenreich und mikroskopisch frei von Kristallen. Ein Erregernachweis aus dem Punktat gelang nicht.

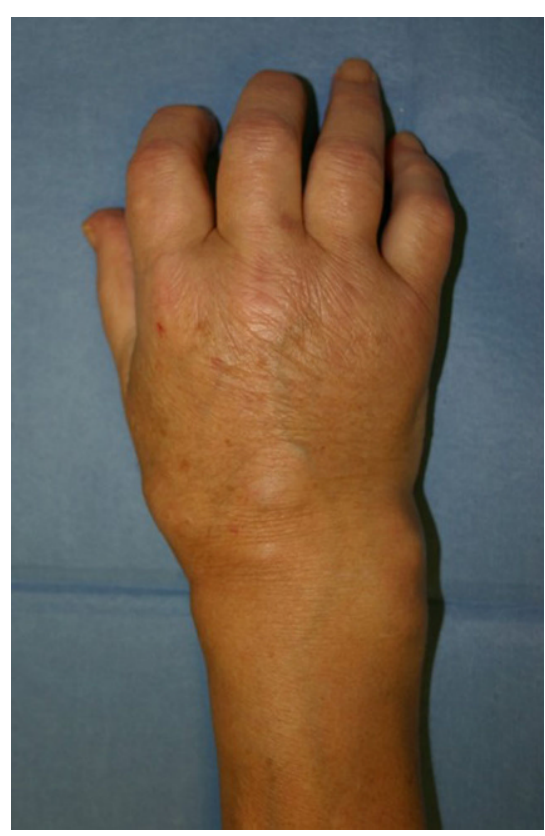

Abb. 1 ॥ Schwellung des rechten Handgelenks mit Ausbreitung über den Handrücken 


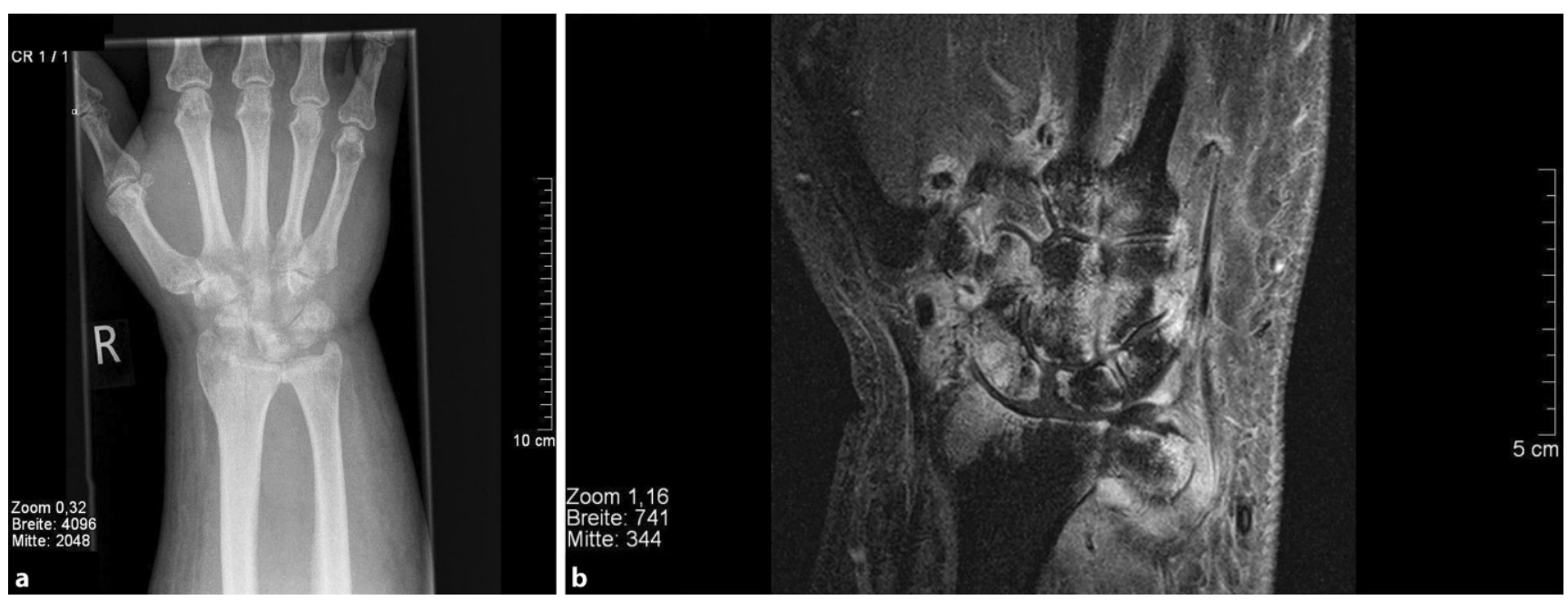

Abb. $2 \Delta$ a Im Röntgenbild Osteitis des distalen Unterarms und des gesamten Carpus mit Erosionen. b Im MRT (T1-Wichtung, fatsat) zusätzlich Synovialitis im Bereich der Strecksehnenfächer

\section{Radiologie}

Im Röntgenbild des Handgelenks in 2 Ebenen und der Magnetresonanztomographie zeigten sich eine Osteitis des distalen Unterarms und gesamten Carpus mit deutlichen Erosionen sowie eine Handgelenksynovialitis. Zusätzlich bestand eine Synovialitis im Bereich der Strecksehnenfächer (• Abb. 2).

\section{Labor und Mikrobiologie}

Die Entzündungsparameter waren unter Einnahme von Prednisolon leicht erhöht: BSG $18 \mathrm{~mm} / \mathrm{h}$, CRP 5,8 mg/l (Referenzbereich bis $5 \mathrm{mg} / \mathrm{l})$, Leukozyten 12,10/nl (Referenzbereich 3,9-10,50/nl).

\section{Weitere Diagnostik}

Die infektiologische Umgebungsdiagnostik auf auslösende Erreger einer reaktiven Arthritis (Yersinien, Chlamydien) blieb ebenso negativ wie die Luesund Borrelienserologie und ein Urethralabstrich auf Gonokokken. Die Bestimmung von HLA-B27, Rheumafaktoren und rheumatologischen Antikörpern war negativ.

\section{Diagnose}

Im Verlauf des stationären Aufenthaltes entwickelte der Patient eine venöse Thrombose der V. jugularis interna, subclavia und axillaris dextra, weshalb der venöse Port explantiert wurde. In den aus dem Portabstrich entnommenen Kulturen zeigte sich ein Wachstum eines mul- tisensiblen P. aeruginosa, sodass bei fehlendem Wachstum in den peripher abgenommen Blutkulturen von einer Besiedelung des venösen Portsystems ausgegangen wurde und eine bakterielle septische Arthritis (SA) vermutet wurde.

\section{Therapie und Verlauf}

Es erfolgte die Synovialektomie des Handgelenks und der Strecksehnenfächer mit gründlichem Débridement, Wundspülung und Drainageneinlage. Auch intraoperativ konnte ein multisensibler $P$. aeruginosa nachgewiesen werden. Im weiteren Verlauf wurden eine Wundrevision und ein Débridement der infizierten Porttasche durchgeführt. Beide Operationen verliefen komplikationslos. Postoperativ erfolgte die kalkulierte antibiotische Therapie mit Cefuroxim und Ciprofloxacin i.v., die nach 3 Tagen resistenzgerecht auf eine Monotherapie mit Ciprofloxacin umgestellt wurde (2mal täglich $750 \mathrm{mg}$ über 7 Tage i.v. und Fortführung über weitere 14 Tage mit 2-mal täglich $500 \mathrm{mg}$ p.o.).

Bei Entlassung aus der stationären Therapie (15 Tage nach der ersten Operation) bestanden reizfreie Wundverhältnisse und Schmerzfreiheit der gesamten Hand. Nach intensiver Handund Physiotherapie gelang eine deutliche Besserung der Funktionseinschränkung. Das Handgelenk zeigte ein Bewegungsausmaß von ROM $85^{\circ}$ in Streckung und Beugung (Extension/Flexion 45-0-40) bei endgradig eingeschränkter Radialund Ulnarduktion. Der Faustschluss aller Finger sowie die Daumenbewegung waren frei. Ein Kompressionshandschuh nach Maß wurde für 3 Monate getragen.

\section{Diskussion}

Wir beschreiben hier den Fall eines Rezidivs einer SA mit $P$. aeruginosa 2 Jahre nach der ersten Episode an unterschiedlicher Lokalisation. In dem beschriebenen Fall war insbesondere die differenzialdiagnostische Abgrenzung zur Arthritis urica schwierig, da anamnestisch und klinisch für beides Hinweise vorlagen. Beide Krankheitsbilder können zudem simultan vorliegen, und ein fehlender Erregernachweis schließt die septische Arthritis nicht aus, wie auch ein fehlender Kristallnachweis die Arthritis urica nicht ausschließt. In unserem Fall machte die fehlende Besserung auf eine Glukokortikoidtherapie die Arthritis urica unwahrscheinlich. Die Entzündungsparameter waren unter Glukokortikoidtherapie nur eingeschränkt verwertbar. Letztlich war der radiologische Nachweis destruierender Veränderungen 10 Tage nach Symptombeginn wegweisend für eine infektiöse Genese. Die Ätiologie der ersten SA lässt sich nicht mehr klären. Unsere Hypothese bezüglich des hier beschriebenen Rezidivs ist, dass es vor oder im Rahmen der ersten Episode zu einer anhaltenden Besiedelung des venösen Portsystems mit $P$. aeruginosa kam, die trotz 
adäquater antibiotischer Therapie nicht eradiziert wurde und ursächlich für die hämatogene Streuung und damit die hier beschriebene Episode der SA des rechten Handgelenks war. Die Symptomfreiheit unseres Patienten zwischen erster SA und Rezidiv lässt vermuten, dass sich auf dem venösen Portsystem ein Biofilm mit $P$. aeruginosa gebildet hat. Elastische Biofilme sind oberflächenassoziierte Bakterienkolonien umgeben von extrazellulärer Matrix und stellen eine gut beschriebene bakterielle Überlebensstrategie dar, die diese vor Antibiotika und Immunantwort des Wirts schützt [2]. Sie bieten ein Reservoir für katheterassoziierte Infektionen [6] und sind eine mögliche Erklärung für die zunehmende Inzidenz medizinproduktassoziierter septischer Arthritiden (SA) [9]. Zu vermuten ist auch, dass die bei vorbestehender Tumorerkrankung mit Chemotherapie bestehende Immunsuppression sowie der vorliegende DM Typ 2 des Patienten eine Biofilmformation begünstigt und/ oder das Auflösen derselben verhindert haben. Im Tiermodell können immunsupprimierte und diabetische Tiere Pseudomonas-aeruginosa-Biofilme schlechter beseitigen als gesunde [13].

Unserem Kenntnisstand nach gibt es keine evidenzbasierten Empfehlungen bezüglich des Umgangs mit langfristigen venösen Verweilkathetern bei $\mathrm{Pa}$ tienten mit SA ohne Hinweis für eine katheterassoziierte Infektion. Der hier beschriebene Fall legt jedoch nahe, dass durch eine frühzeitigere Entfernung des venösen Portsystems das Rezidiv hätte vermieden werden können.

Die SA geht, abhängig von ambulantem oder nosokomialem Erwerb der Infektion, Alter und betroffenem Gelenk mit einer 30-Tages-Mortalität von bis zu $26 \%$ und einem Funktionsverlust des Gelenks in bis zu $50 \%$ einher $[4,10]$. Eine unverzügliche Diagnostik und Therapie ist prognoseentscheidend. Die Infektion des Gelenks wird entweder durch hämatogene Streuung, direkte Inokulation im Rahmen eines Traumas mit lokaler oder regionaler Fortleitung oder iatrogen hervorgerufen. Generelle Risikofaktoren für die SA sind Alter $>80$ Jahre, Diabetes mellitus, HIV, rheumatoide Arthritis, bestehende Gelenkendoprothesen oder

Z Rheumatol 2020 · 79:906-909 https://doi.org/10.1007/s00393-020-00842-y

(c) Der/die Autor(en) 2020

C. Thibeault · U. Schneider · A. Eisenschenk · M. Lautenbach

Rezidiv einer septischen Arthritis des Handgelenks durch Pseudomonas aeruginosa bei Besiedelung eines zentralvenösen Verweilkatheters

Zusammenfassung

Ein Diabetiker, Träger eines Ports und mit Zustand nach Gonarthritis durch Pseudomonas aeruginosa, erlitt eine subakute Arthritis eines Handgelenks. Protrahiert gelang der kulturelle Nachweis von $P$. aeruginosa aus dem explantierten Port und dem betroffenen Gelenk. Der Fall zeigt, dass bei Patienten mit unklarer Handgelenkarthritis, Vorgeschichte einer septischen Arthritis mit $P$. aeruginosa und Risikofaktoren für eine hämatogene Streuung ein Rezidiv ausgeschlossen werden sollte. Die Therapie bestand aus Portexplantation, Débridement mit Synovialektomie des Gelenks und antibiotischer Therapie.

\section{Schlüsselwörter}

Septische Arthritis · Infektiöse Arthritis . Bakterielle Arthritis · Monoarthritis · Biofilm

\section{Recurrence of septic arthritis of the wrist by Pseudomonas aeruginosa due to colonization of a central venous indwelling catheter}

\section{Abstract}

A diabetic patient, wearer of a port and with a history of gonarthritis due to Pseudomonas aeruginosa presented with subacute arthritis of a wrist. After a protracted interval $P$. aeruginosa was detected by microbial culture from the explanted port and the affected joint. This case shows that in patients with unclear arthritis of the wrist, a history of septic arthritis with P. aeruginosa and risk factors for hematogenous spread, a recurrent infection should be excluded. The treatment consisted of explantation of the port, débridement with synovectomy of the joint and adequate antibiotic treatment.

Keywords

Septic arthritis · Infectious arthritis · Bacterial arthritis · Monoarthritis · Biofilm vorausgegangene Gelenkoperationen und Hautinfektionen [3, 7]. Ein erhöhtes Risiko für eine hämatogene Ursache einer septischen Arthritis haben immunsupprimierte und hospitalisierte Patienten, insbesondere wenn bei diesen Gefäßkatheter oder größere invasive Eingriffe notwendig waren [8].

Das typische klinische Bild der septischen Arthritis ist eine akute Monoarthritis. Häufige Differenzialdiagnosen der akuten Monoarthritis sind neben der Infektion durch Bakterien, Pilze oder Mykobakterien unter anderem reaktive Arthritiden, Traumafolgen, Kristallopathien, rheumatoide Arthritiden, Spondyloarthritiden und Kollagenosen [7]. Die klinischen Zeichen mit der höchsten Sensitivität für eine bakterielle SA sind eine Schmerzexazerbation bei Bewegung, Rötungen und Schwellungen sowie ein Gelenkerguss. Die größte diagnostische Vorhersagekraft hat ein leukozytenrei- ches Gelenkpunktat. Laborparameter wie CRP-, Procalcitoninwert, periphere Leukozytenzahl und BSG haben aufgrund von niedriger Sensitivität und/oder Spezifität nur in der Zusammenschau der Befunde einen diagnostischen Nutzen [1]. Die Diagnose der bakteriellen SA beruht auf der mikrobiellen Erregerkultur aus dem Gelenk oder bei typischem klinischem Bild auf dem Erregernachweis in Blutkulturen, wenn kein Keimnachweis im Gelenkpunktat zu erlangen ist [8]. Die Infektion ist oft monomikrobiell. Staphylococcus aureus ist der häufigste auslösende Erreger (je nach Literaturangabe, Ursache der SA und betroffenem Gelenk bis $95 \%)$. P. aeruginosa ist ein gramnegativer Nonfermenter und ein seltener Auslöser der bakteriellen SA (3-4\%), der meist nosokomial, oft im Zusammenhang mit invasiven Maßnahmen erworben wird [5, 10, 11]. Einzelne Fälle von rezidivierenden SA mit Pseudomonas aeruginosa sind 
beschrieben [12]. Die Therapie der SA beruht auf dem arthroskopischen oder offen chirurgischen Débridement mit Synovialektomie des betroffenen Gelenks sowie einer resistenzgerechten systemischen Antibiotikatherapie.

\section{Fazit für die Praxis}

- Bei unklarer Monoarthritis, bakterieller septischer Arthritis in der Vorgeschichte und prädisponierenden Faktoren für eine hämatogene Streuung muss ein Rezidiv ausgeschlossen werden.

- Die frühzeitige Diagnose der SA ist prognoseentscheidend. Ein Keimnachweis sollte unbedingt durch Punktion oder ggf. chirurgisch angestrebt werden.

- Die Mortalität und Morbidität der SA ist unbehandelt hoch.

- Die Therapie erfolgt chirurgisch und antimikrobiell nach Resistogramm.

- Portsysteme können durch Biofilme besiedelt werden. Bei Nachweis einer bakteriellen SA sollte eine Explantation erwogen werden, um Rezidive zu verhindern.

\section{Korrespondenzadresse}

\section{Dr. med. C. Thibeault}

Medizinische Klinik mit Schwerpunkt Infektiologie und Pneumologie, Charité Universitätsmedizin Berlin, Campus Virchow Klinikum

Augustenburger Platz 1, 13353 Berlin, Deutschland

charlotte.thibeault@charite.de

Funding. Open Access funding provided by Projekt DEAL.

\section{Einhaltung ethischer Richtlinien}

Interessenkonflikt. C. Thibeault, U. Schneider, A. Eisenschenk und M. Lautenbach geben an, dass kein Interessenkonflikt besteht.

Für diesen Beitrag wurden von den Autoren keine Studien an Menschen oder Tieren durchgeführt. Für die aufgeführten Studien gelten die jeweils dort angegebenen ethischen Richtlinien. Für Bildmaterial oder anderweitige Angaben innerhalb des Manuskripts, über die Patienten zu identifizieren sind, liegt von ihnen und/oder ihren gesetzlichen Vertretern eine schriftliche Einwilligung vor.
Open Access. Dieser Artikel wird unter der Creative Commons Namensnennung 4.0 International Lizenz veröffentlicht, welche die Nutzung, Vervielfältigung, Bearbeitung, Verbreitung und Wiedergabe in jeglichem Medium und Format erlaubt, sofern Sie den/die ursprünglichen Autor(en) und die Quelle ordnungsgemäß nennen, einen Link zur Creative Commons Lizenz beifügen und angeben, ob Änderungen vorgenommen wurden.

Die in diesem Artikel enthaltenen Bilder und sonstiges Drittmaterial unterliegen ebenfalls der genannten Creative Commons Lizenz, sofern sich aus der Abbildungslegende nichts anderes ergibt. Sofern das betreffende Material nicht unter der genannten Creative Commons Lizenz steht und die betreffende Handlung nicht nach gesetzlichen Vorschriften erlaubt ist, ist für die oben aufgeführten Weiterverwendungen des Materials die Einwilligung des jeweiligen Rechteinhabers einzuholen.

Weitere Details zur Lizenz entnehmen Sie bitte der Lizenzinformation auf http://creativecommons.org/ licenses/by/4.0/deed.de.

\section{Literatur}

1. Carpenter CR, Schuur JD, Everett WW, Pines JM (2011) Evidence-based diagnostics: adult septic arthritis. Acad Emerg Med 18(8):782-796. https:// doi.org/10.1111/j.1553-2712.2011.01121.x

2. Hall-Stoodley L, Stoodley P (2009) Evolving concepts in biofilm infections. Cell Microbiol 11:1034-1043. https://doi.org/10.1111/j.1462 5822.2009.01323.x

3. Kaandorp CJE, Van Schaardenburg D, Krijnen $P$, Habbema JDF, Van De Laar MAFJ (1995) Risk factors for septic arthritis in patients with joint disease. Arthritis Rheum 38(12):1819-1825. https://doi. org/10.1002/art.1780381215

4. Kaandorp CJE, Krijnen P, Moens HJB, Habbema JDF, van Schaardenburg D (1997) The outcome of bacterial arthritis. A prospective communitybased study. Arthritis Rheum 40(5):884-892 https://doi.org/10.1002/art.1780400516

5. Lepetit C, Le Gal S, Michon J, Collon S, Tillou X (2015) Pseudomonas aeruginosa septic trapezometacarpal arthritis after prostate laser vaporization. Infection 43(4):503-505. https://doi.org/10. 1007/s15010-015-0729-2

6. Maki DG, Kluger DM, Crnich CJ (2006) The risk of bloodstream infection in adults with different Intravascular devices: a systematic review of 200 published prospective studies. Mayo Clin Proc 81(9):1159-1171. https://doi.org/10.4065/81.9. 1159

7. Margaretten ME, Kohlwes J, Moore D, Bent S (2007) Does this adult patient have septic arthritis? JAMA 297(13):1478-1488

8. Mathews CJ, Weston VC, Jones A, Field M Coakley G (2010) Bacterial septic arthritis in adults. Lancet 375(9717):846-855. https://doi.org/10. 1016/S0140-6736(09)61595-6

9. Murillo O, Grau I, Lora-Tamayo J (2015) The changing epidemiology of bacteraemic osteoarticular infections in the early 21st century. Clin Microbio Infect 21(3):254.e1-254.e8. https://doi.org/10. 1016/j.cmi.2014.09.007

10. Murillo O, Gomez-Junyent J, Grau I (2016) Clinical findings of bacteremic septic arthritis according to the site of acquisition: The overlap between health care-related and community- and nosocomial- acquired cases. Eur J Intern Med 28:38-42. https:// doi.org/10.1016/j.ejim.2015.11.013

11. Titécat $M$, Senneville E, Wallet F (2013) Bacterial epidemiology of osteoarticular infections in a referent center: 10-year study. Orthop Traumatol Surg Res 99(6):653-658. https://doi.org/10.1016/j. otsr.2013.02.011

12. Vickers MH, Price T (1993) Recurrent pseudomonas septic arthritis. Postgrad Med J 69(818):950-951. https://doi.org/10.1136/pgmj.69.818.950

13. Watters C, DeLeon K, TrivediU (2013) Pseudomonas aeruginosa biofilms perturb wound resolution and antibiotic tolerance in diabetic mice. Med Microbiol Immunol 202(2):131-141. https://doi. org/10.1007/s00430-012-0277-7 